\title{
Article
}

\section{Infrared Spectroscopy Coupled with a Dispersion Model for Quantifying the Real-Time Dynamics of Kanamycin Resistance in Artificial Microbiota}

Jin, Naifu, Paraskevaidi, Maria, Semple, Kirk T., Martin, Francis L and Zhang, Dayi

Available at https://clok.uclan.ac.uk/19808/

Jin, Naifu, Paraskevaidi, Maria, Semple, Kirk T., Martin, Francis L orcid iconORCID: 0000-0001-8562-4944 and Zhang, Dayi (2017) Infrared Spectroscopy Coupled with a Dispersion Model for Quantifying the Real-Time Dynamics of Kanamycin Resistance in Artificial Microbiota. Analytical Chemistry, 89 (18). pp. 9814-9821. ISSN 0003-2700

It is advisable to refer to the publisher's version if you intend to cite from the work. http://dx.doi.org/10.1021/acs.analchem.7b01765

For more information about UCLan's research in this area go to http://www.uclan.ac.uk/researchgroups/ and search for <name of research Group>.

For information about Research generally at UCLan please go to http://www.uclan.ac.uk/research/

All outputs in CLoK are protected by Intellectual Property Rights law, including Copyright law. Copyright, IPR and Moral Rights for the works on this site are retained by the individual authors and/or other copyright owners. Terms and conditions for use of this material are defined in the policies page. 

10 11 12

Infrared spectroscopy coupled with a dispersion model for quantifying the real-time dynamics of kanamycin resistance in artificial microbiota

Naifu Jin ${ }^{1}$, Maria Paraskevaidi ${ }^{2}$, Kirk T. Semple $^{1}$, Francis L. Martin ${ }^{2, *}$, Dayi Zhang, ${ }^{1, *}$

${ }^{1}$ Lancaster Environment Centre, Lancaster University, Lancaster, LA1 4YQ, UK;

${ }^{2}$ School of Pharmacy and Biomedical Sciences, University of Central Lancashire, Preston PR1 2HE, UK

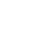

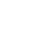

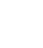

\section{*Corresponding authors:}

Dayi Zhang, Lancaster Environment Centre, Lancaster University, Lancaster LA1 4YQ, UK; Tel.: +44(0)1524 510288; Fax: +44(0)1524 510082, Email:

\section{d.zhang@lancaster.ac.uk}

Francis L. Martin, School of Pharmacy and Biomedical Sciences, University of Central Lancashire, Preston PR1 2HE, UK; Tel.: +44(0)1772 896482; Email:

\section{flmartin@uclan.ac.uk}




\section{Abstract}

Over-usage of antibiotics leads to the widespread induction of antibiotic resistance genes (ARGs). Developing an approach to allow real-time monitoring and fast prediction of ARGs dynamics in clinical or environmental samples has become an urgent matter. Vibrational spectroscopy is potentially an ideal technique towards the characterization of the microbial composition of microbiota as it is non-destructive, high-throughput and label-free. Herein, we employed attenuated total reflection Fourier-transform infrared (ATR-FTIR) spectroscopy and developed a spectrochemical tool to quantify the static and dynamic composition of kanamycin resistance in artificial microbiota to evaluate microbial antibiotic resistance. Second order differentiation was introduced in identifying the spectral biomarkers, and principal component analysis followed by linear discriminant analysis (PCA-LDA) was used for the multivariate analysis of the entire spectral features employed. The calculated results of the mathematical dispersion model coupled with PCA-LDA showed high similarity to the designed microbiota structure, with no significant difference $(P>0.05)$ in the static treatments. Moreover, our model successfully predicted the dynamics of kanamycin resistance within artificial microbiota under kanamycin pressures. This work lends new insights into the potential role of spectrochemical analyses in investigating the existence and trends of antibiotic resistance in microbiota.

Keywords Antibiotic resistance, Artificial microbiota, ATR-FTIR spectroscopy, Kanamycin, Multivariate analysis, Spectrochemical 


\section{Introduction}

Antibiotics have played a vital role in modern medicine contributing to a considerable reduction in childhood mortality and increasing life expectancy ${ }^{1}$. However, the increasing number of fatal infections caused by antibiotic-resistant bacteria is gradually developing into a global threat. The environment has become the primary "sink" for most applied antibiotics and their residues arising from human or animal excretion $^{1-3}$. Since bacteria with antibiotic resistance genes (ARGs) can tolerate antibiotics, selection pressures from contaminated water or soil will boost the abundance of ARGs in the environment and increase the possibility of their spread through microbial species ${ }^{4,5}$. Therefore, real-time monitoring and quantification of ARGs or antibiotic-resistant bacteria is urgently required.

Besides measuring the concentration of antibiotics via chemical analysis, various biological analytical methods have been used to determine the presence, abundance and diversity of ARGs in the microbiota to capture a "static map" of their existence, e.g., meta-sequence and quantitative polymerase chain reaction $(\mathrm{qPCR})^{6,7}$. However, genetically identical cells from the same population have stochasticity in gene expression, meaning that there is significant variation in their molecular content and phenotype, even under similar environmental influences. Moreover, bacterial resistance to the antibiotics can also be affected and regulated epigenetically ${ }^{8}$. In combination, these factors provide an opportunity for phenotypic and cell-type diversity regardless of genotype ${ }^{9}$. This questions the reliability of determining ARGs abundance by molecular biological approaches in real-world situations, leading to the necessity of developing a phenotypic assay that depicts in situ dynamics of ARGs or microbial antibiotic resistance in environmental samples.

It is well accepted that genetic and epigenetic factors cannot be studied independently as a complete phenotype emerges from both together ${ }^{10}$. The spectrochemical analysis is an alternative approach to characterize the phenotypic features of organisms and has already demonstrated its ability to investigate clinical 
samples, as well as to describe and identify bacterial species ${ }^{11,12}$. Previous studies indicates that spectroscopic techniques are capable of studying phenotypic features, at either population ${ }^{13}$ or single-cell ${ }^{14}$ level, such as diagnosing the distinct spectral signatures and metabolomes from isogenic cell lines ${ }^{15}$. However, the current techniques have limited application in characterizing ARGs under antibiotic pressures, mainly due to the lack of appropriate analytical models and well-trained databases. Recently, some studies using spectroscopic techniques have set out to investigate biological response to environmental stress, like nanomaterials ${ }^{16,17}$ and antimicrobial reagents ${ }^{18,19}$. The introduction of spectroscopic techniques coupled with a suitable prediction model to characterize microbial composition may bring new insights in detecting the presence or even the dynamics of microbial antibiotic resistance in environmental microbiota in real-time, owing to its non-destructive, high-throughput and label-free character ${ }^{20,21}$. It also allows for in situ spectral measurements, helping in understanding the interactions between microbes and their physical environment.

Kanamycin is a subclass of aminoglycoside antibiotics, one of the most widely applied antibiotics in health and molecular biology $y^{22}$. Because of the well-established mechanisms of kanamycin resistance and characterized sequence ${ }^{23}$, it was selected as the model antibiotic in the present study. Herein, we used attenuated total reflection Fourier-transform infrared (ATR-FTIR) spectroscopy, coupled with the multivariate analysis and the dispersion indicator model, to quantify the kanamycin resistance within artificial microbiota and evaluate their phenotypic change associated with kanamycin resistance, from both static and dynamic perspectives. This work raises the potential feasibility of applying spectroscopic techniques to diagnose ARGs phenotypic dynamics in the microbial community in situ.

\section{Experimental section}

\section{Sample preparation}

The present study included two strains without kanamycin-resistant-gene, Mycobacterium vanbaalenii PYR-1 and Escherichia coli DH5 $\alpha$, and one 
kanamycin-resistant strain Acinetobacter baylyi ADPWH_recA, which has a continuously expressed kanamycin resistance gene $\mathrm{kan}^{R}$ (from Mini-Tn $5 / \mathrm{Km}^{24}$, Genbank accession number: U32991.1) inserted into the recA gene in the chromosome of $A$. baylyi $\mathrm{ADP} 1^{25}$. Before the experiment, they were all cultured in Luria-Bertani (LB) broth medium for $24 \mathrm{~h}$ at $30 \pm 2^{\circ} \mathrm{C}$.

The three control groups contained pure M. vanbaalenii PYR-1, E. coli DH5a and A. baylyi ADPWH_recA, respectively. The artificial microbiotas were prepared for both static (M1 to M5) and dynamic (AM1 and AM2) experiments by gently mixing the cells in the compositions listed in Table 1 . The optical density at $600 \mathrm{~nm}$ $\left(\mathrm{OD}_{600}\right)$ in each treatment was monitored continuously for $24 \mathrm{~h}$ by a multimode plate reader (FLUOstar Omega, Germany) to evaluate bacterial growth. For static tests, the cells were directly collected by centrifugation ( $4000 \mathrm{rpm}$ for $5 \mathrm{~min}$ ), washed three times with sterile deionized water to remove the residues of growth media and then suspended in $70 \%$ ethanol to fix the bacterial cells. For dynamic tests, all the artificial microbiotas were treated with kanamycin (final concentration $10 \mathrm{mg} / \mathrm{L}$ ). After exposure for 4, 8, 12 or $24 \mathrm{~h}$, the cells from microbiotas were harvested following the same procedure as above.

Table 1. The compositions of artificial microbiotas (volume ratio, v:v:v).

\begin{tabular}{|c|c|c|c|c|c|c|c|c|c|c|}
\hline \multirow[b]{2}{*}{ Treatments } & \multicolumn{3}{|c|}{ Control } & \multicolumn{5}{|c|}{ Static test } & \multicolumn{2}{|c|}{ Dynamic test } \\
\hline & $\begin{array}{c}\text { M. } \\
\text { vanbaalenii }\end{array}$ & $\begin{array}{c}E . \\
\text { coli }\end{array}$ & $\begin{array}{c}\text { A. } \\
\text { baylyi }\end{array}$ & $\mathbf{M}_{1}$ & $\mathbf{M}_{2}$ & $\mathbf{M}_{3}$ & $\mathbf{M}_{4}$ & $\mathbf{M}_{5}$ & $\mathbf{A M}_{1}$ & $\mathbf{A M}_{2}$ \\
\hline M. vanbaalenii & $100 \%$ & - & - & $40 \%$ & $30 \%$ & $30 \%$ & $15 \%$ & $5 \%$ & $40 \%$ & $25 \%$ \\
\hline E. coli & - & $100 \%$ & - & $50 \%$ & $45 \%$ & $20 \%$ & $10 \%$ & $5 \%$ & $40 \%$ & $25 \%$ \\
\hline A. baylyi & - & - & $100 \%$ & $10 \%$ & $25 \%$ & $50 \%$ & $75 \%$ & $90 \%$ & $20 \%$ & $50 \%$ \\
\hline
\end{tabular}

\section{ATR-FTIR spectroscopy}

The washed cell pellets (minimal amount $>5 \mu \mathrm{L}$ ) were applied onto Low-E slides for the interrogation by ATR-FTIR spectroscopy. A TENSOR 27 FTIR spectrometer (Bruker Optics Ltd., UK) equipped with a Helios ATR attachment (containing a 
diamond internal IRE; incidence angle of the IR beam: $45^{\circ}$ ) was used. Instrument parameters were set at 32 scans and spatial resolution of $8 \mathrm{~cm}^{-1}$. Before the measurement of a new sample, the crystal was cleaned with deionized water and background readings were retaken. A total of 30 spectra were randomly acquired for each treatment (3 replicates).

\section{Computational analysis}

The primary analysis methods employed in this study involved multivariate analysis and the dispersion indicator model. The initial data generated from ATR-FTIR spectroscopy were analyzed within MATLAB R2011a (TheMathsWorks, Natick, MA, USA) software, coupled with IRootLab toolbox (http://irootlab.googlecode.com) ${ }^{26}$. Unless otherwise stated, the acquired spectra were cut to the biochemical-cell fingerprint region (1800-900 $\left.\mathrm{cm}^{-1}\right)$, rubberband baseline corrected and normalized to Amide I $\left(1650 \mathrm{~cm}^{-1}\right)$. Second order differentiation baseline correction and vector normalization was also performed as an alternative mean to process the data (the number of the filter coefficients of the Savitzky-Golay smoothing/differentiation filter was 9). Principal component analysis followed by linear discriminant analysis (PCA-LDA) was subsequently applied to the pre-processed data to reduce the number of spectra to 10 uncorrelated principal components (PCs), which account for $>99 \%$ of the total variance; LDA is a supervised technique coupled with PCA in order to maximize inter-class and minimize intra-class variance ${ }^{21}$. In addition, cluster vector approach was conducted to visualise the discriminating difference ${ }^{21,27}$. This method takes input from PCA-LDA to create a loadings vector for each category contributing to respective data points. The pseudo-spectra allow identifying which variables (or wavenumber) are responsible for variance in the data set related to the original spectra $^{21,27}$. The detailed information of the dispersion indicator model was described in the Electronic Supplementary Information (ESI).

\section{Biological analysis}

The copy numbers of total bacterial $16 \mathrm{~S}$ rRNA and targeted kanamycin resistance gene $\left(\mathrm{kan}^{R}\right)$ were determined by quantitative polymerase chain reaction (qPCR). For 16S rRNA, the primer pair set was 341F (5'-CCTACGGGNGGCWGCAG-3') and 805R (5'-GACTACHVGGGTATCTAATCC-3'), and the primer pair for $k^{\prime}{ }^{R}$ was KanF (5'-TGTCATACCACTTGTCCGCC-3') and KanR 
(5'-ATCGAGCTGTATGCGGAGTG-3'). The $20 \mu \mathrm{L}$ qPCR system consisted of $2 \mu \mathrm{L}$ of each primer, $1 \mu \mathrm{L}$ DNA template, $5 \mu \mathrm{L}$ molecular water and $10 \mu \mathrm{L} \mathrm{iTaq}^{\mathrm{TM}}$ Universal SYBR ${ }^{\circ}$ Green Supermix (BioRad, USA). The relative abundance of kanR in each pure strain was calculated as the ratio of kanR copy numbers to $16 \mathrm{~S}$ rRNA copy numbers $(\mathrm{kanR} / 16 \mathrm{~S})$. The microbial kanamycin resistance within the artificial microbiota was calculated as the ratio of $A$. baylyi population to the total bacterial population.

\section{Statistical analysis}

One-way analysis of variance (ANOVA) with Tukey's post hoc test/or T-test was employed to examine the discriminating differences. All statistical analysis was carried out in GraphPad Prism 6.

\section{Results and Discussion}

\section{Growth and kanamycin resistance gene of individual strains}

All the three bacterial strains (A. baylyi ADPWH_recA, M. vanbaalenii PYR-1 and E. coli $\mathrm{DH} 5 \alpha$ ) had similar growth curves without kanamycin pressure (see ESI Figure S1A). Cultivated in $10 \mathrm{mg} / \mathrm{L}$ kanamycin, only A. baylyi ADPWH_recA maintained positive growth because of the expression of $\mathrm{kan}^{R}$ gene and resistance to kanamycin (see ESI, Figure S1B). Neither M. vanbaalenii PYR-1 nor E. coli DH5a grew post-exposure to $10 \mathrm{mg} / \mathrm{L}$ kanamycin. The results of qPCR further confirmed that the high relative abundance of $\mathrm{kan}^{R}$ gene (kanR/16S) were only found in $A$. baylyi ADPWH_recA (0.306 in medium without kanamycin and 0.275 in medium with 10 $\mathrm{mg} / \mathrm{L}$ kanamycin respectively, no significant difference), whereas it was less than 0.001 or below the limit of detection for M. vanbaalenii PYR-1 or E. coli DH5a (see ESI, Figure S2). It was further proved that kanamycin resistance gene is only detectable in A. baylyi ADPWH_recA, but neither M. vanbaalenii PYR-1 nor E. coli DH5 $\alpha$, and the latter two cannot tolerate kanamycin pressure. The active group of kanamycin, 2-deoxystreptamine, impairs bacterial protein synthesis through binding to prokaryotic ribosomes $30 \mathrm{~S}$ subunit ${ }^{22}$. The $\mathrm{kan}^{R}$ encoding neomycin phosphotransferase is an aminoglycoside-modifying enzyme, using ATP as donor to modify the hydroxyl functions of 2-deoxystreptamine and inhibit its binding to 
ribosomes ${ }^{28}$. The $\mathrm{kan}^{R}$ gene is therefore a reliable molecular indicator in detecting the kanamycin resistance.

\section{IR spectral fingerprints of individual strains and microbiotas}

The IR spectral fingerprint region $\left(1800-900 \mathrm{~cm}^{-1}\right)$ of the three strains and artificial microbiotas are shown in Figure 1. The representative peaks of the biochemical fingerprint include lipids $\left(\sim 1750 \mathrm{~cm}^{-1}\right)$, Amide I $\left(\sim 1650 \mathrm{~cm}^{-1}\right)$, Amide II $\left(\sim 1550 \mathrm{~cm}^{-1}\right)$, Amide III $\left(\sim 1260 \mathrm{~cm}^{-1}\right)$, carbohydrate $\left(\sim 1155 \mathrm{~cm}^{-1}\right)$, asymmetric phosphate stretching vibrations $\left(v_{\mathrm{as}} \mathrm{PO}_{2}^{-} ; \sim 1225 \mathrm{~cm}^{-1}\right)$, symmetric phosphate stretching vibrations $\left(v_{\mathrm{s}} \mathrm{PO}_{2}{ }^{-}\right.$; $\left.\sim 1080 \mathrm{~cm}^{-1}\right)$, glycogen $\left(\sim 1030 \mathrm{~cm}^{-1}\right)$ and protein phosphorylation $\left(\sim 970 \mathrm{~cm}^{-1}\right)^{2021}$. Past literatures ${ }^{12,20,29,30}$ suggest the characteristic peaks given by the region can be used as biomarkers to characterize microbial cell types (even at subspecies level) and diagnose microbe-induced diseases.

However, the visual spectral differences with the mean spectra are almost identical regardless of the bacterial species or community composition. For this reason, we applied the cluster vectors after multivariate analysis (PCA-LDA) and the second order differentiation baseline correction to further reveal the underlying biochemical differences between each strain or microbiota. Based on the derived spectral biomarkers from PCA-LDA (Figure 1B), all the microbiota samples showed marked segregation (see ESI, Table S1). Characteristics associated with microbial composition were observed in particular wavenumber-absorbance intensities. For instance, the intensities at $980 \mathrm{~cm}^{-1}$ and $1740 \mathrm{~cm}^{-1}$ were increased with increasing ratio of ARGs but fluctuated in some artificial microbiotas, particularly for microbiota M3 (M. vanbaalenii PYR-1: E. coli DH5 $\alpha$ : A. baylyi ADPWH_recA = 30\%:20\%:50\%). Additionally, IR spectral analysis (Figure 2A) based on the second order differentiation baseline correction and vector normalization highlighted several key biomarkers. Two apparent shifts from $\sim 1630 \mathrm{~cm}^{-1}$ to $\sim 1640 \mathrm{~cm}^{-1}$ (Amide I) and from $\sim 1222 \mathrm{~cm}^{-1}$ to $\sim 1235 \mathrm{~cm}^{-1}\left(v_{\mathrm{as}} \mathrm{PO}_{2}{ }^{-}\right)$associated with $A$. baylyi were regarded as biomarkers for the presence of kanamycin resistance. These spectral alterations might be attributed to the upregulated activities of the $\mathrm{kan}^{R}$ encoding aminoglycoside $O$-phosphotransferase, which contributes to microbial resistance by inactivating kanamycin molecular via catalyzing ATP-dependent phosphorylation of specific aminoglycoside hydroxyl groups ${ }^{31}$. Some other weaker discriminations included the 
221 polysaccharide fingerprint region $\left(1000-1150 \mathrm{~cm}^{-1}\right)$ and the protein absorbance region $222\left(1500-1700 \mathrm{~cm}^{-1}\right)^{27}$. These alterations were probably induced by the interference of 223 extracellular polymeric substances (EPS) produced by different species ${ }^{32-34}$ and resulted in the difficulties in distinguishing biomarkers from the PCA-LDA extracted peaks. Based on the previous studies ${ }^{32,35,36}$, we speculate that these extracellular materials may interact with each other and generate new biochemical compositions within the communities, influencing the discriminating peaks obtained with spectrochemical interrogation.
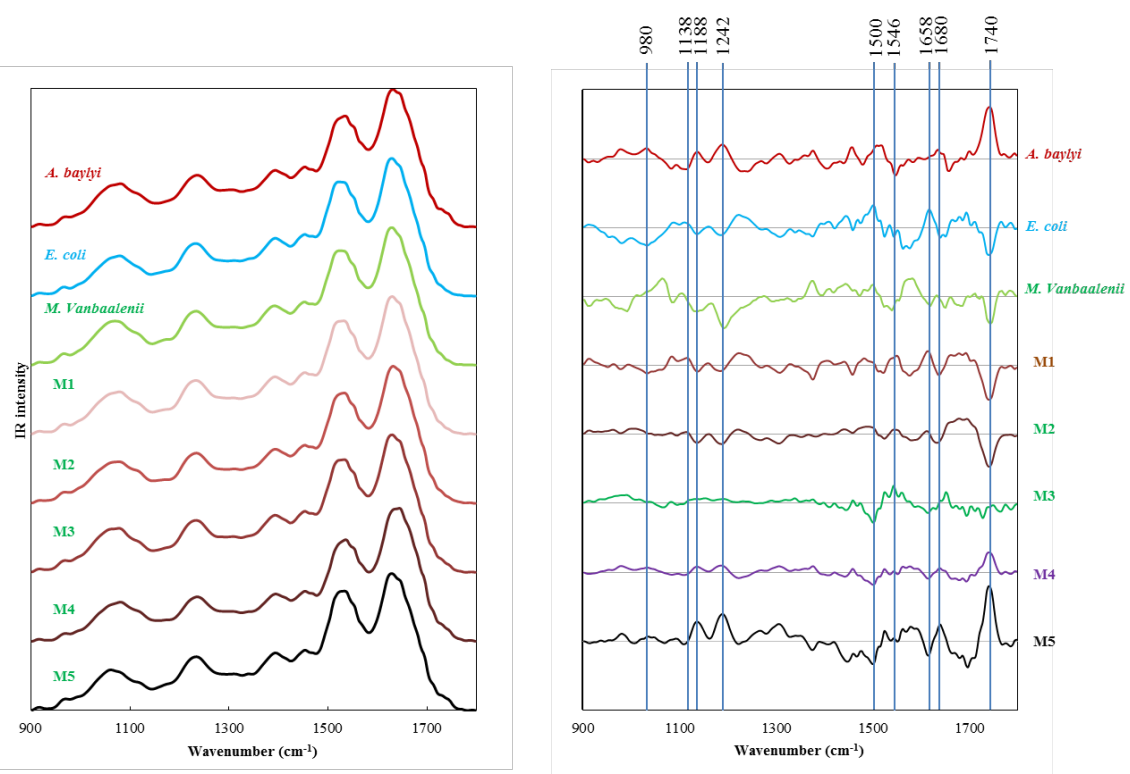

231 Figure 1. (A) Infrared spectra of A. baylyi, M. vanbaalenii, E. coli and five artificial microbiotas (M1-M5). (B) Cluster vector plots after PCA-LDA, indicating significant wavenumbers for the segregation between bacterial species and artificial microbiotas. 


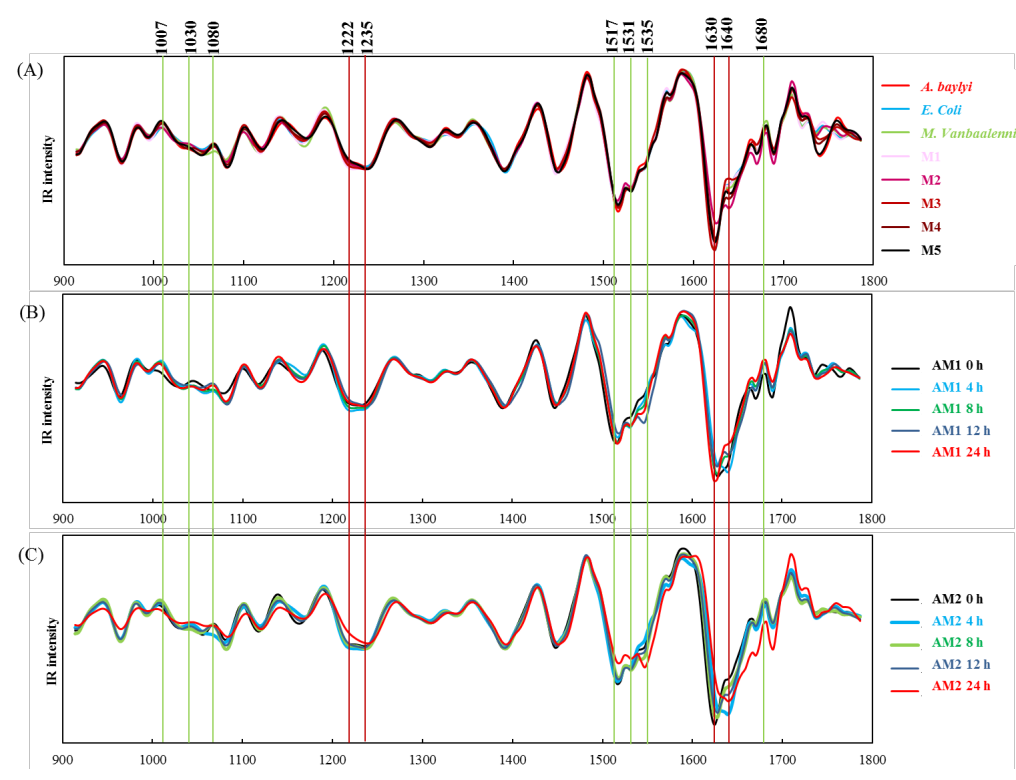

Figure 2. Class means spectra of pre-processed data based on second order differentiation baseline correction and vector normalization. (A) Processed spectra of A. baylyi, M. vanbaalenii, E. coli and five artificial microbiotas (M1-M5). Processed spectra of AM1 at different time point in dynamic experiment. (C) Processed spectra of AM1 at different time point in dynamic experiment.

\section{Predicting community composition in artificial microbiotas}

Comparing to the IR spectra in the static tests, we observed identical spectral biomarkers in artificial community dynamics (Figure $2 \mathrm{~B}$ and $2 \mathrm{C}$ ) that the same shifts from $\sim 1630 \mathrm{~cm}^{-1}$ to $\sim 1640 \mathrm{~cm}^{-1}$ (Amide I) and from $\sim 1222 \mathrm{~cm}^{-1}$ to $\sim 1235 \mathrm{~cm}^{-1}$ $\left(v_{\mathrm{as}} \mathrm{PO}_{2}^{-}\right)$developed along with the time. The results indicated the consistent spectral biomarkers in both static and dynamic microbiotas in analyzing the phenotypic presence and abundance of kanamycin resistance gene in the targeted microbiota.

The PCA-LDA scores plot (Figure 3A) also illustrates a significant segregation of the different groups, associated with differing microbiota compositions. The control groups (M. vanbaalenii, E. coli, and A. baylyi) are clearly separated from each other. In contrast to $M$. vanbaalenii and E. coli, all the converted spectral values of $A$. baylyi are aligned as negative along linear discriminant one (LD1), likely attributed to its kanamycin resistance. Meanwhile, along with linear discriminant two (LD2), the group of M. vanbaalenii (Gram-positive bacteria) is located on the negative axis alone, separated from the other two groups (E. coli and A. baylyi), which are Gram-negative. 
The five artificial microbiota samples (M1 to M5) are located inbetween, and their distances to the control groups are correlated with their community compositions.
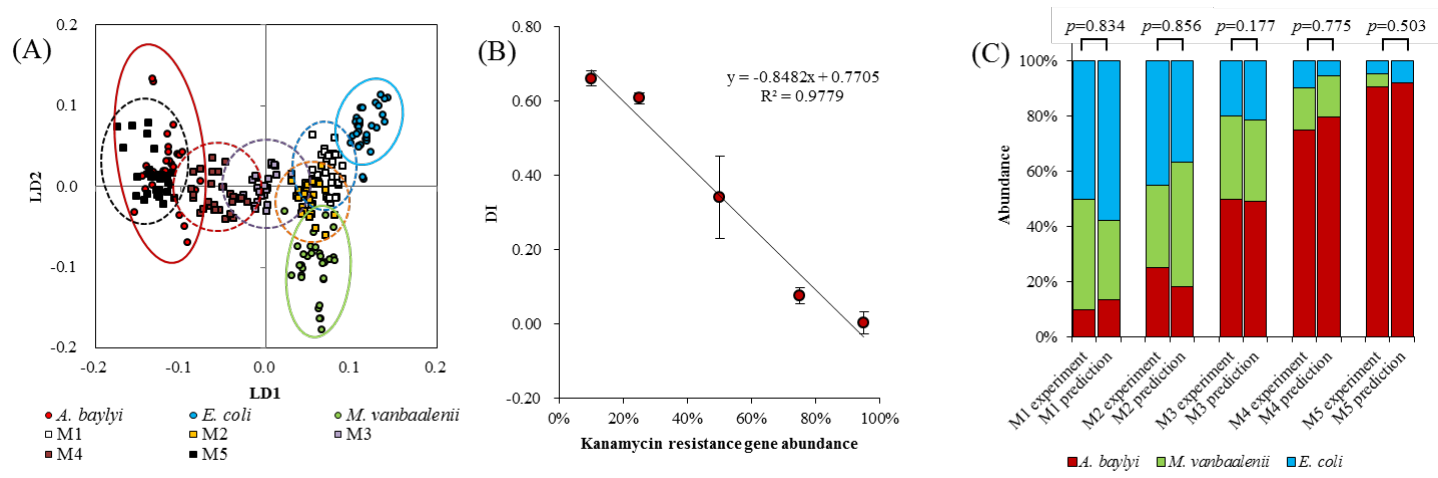

Figure 3. (A) Two-dimensional (LD1, LD2) scores plot after PCA-LDA of pure microbial strains and artificial microbiotas with different composition. (B) Correlation between kanamycin resistance gene abundance and group distance dispersion $\left(D_{I}\right)$. (C) Comparison of artificial microbiota composition between experimental data and model prediction.

In order to predict the composition of artificial microbiota, the dispersion indicator mode ${ }^{37}$ was carried out by transferring the dispersion analysis from the IR spectral variables to the vectors (LD1 and LD2) and using $D_{I}$ as the indicator, comparing to the ARGs gene copy numbers quantified by qPCR as reference. This method used the summarized spectral information from PCA-LDA which accounts for over $90 \%$ of spectral variations in the present study, and was more conclusive than the limited biomarkers from second order differentiation. Here, microbiotas with less abundance of $A$. baylyi were further separated from the A. baylyi group, but closer to those of $E$. coli and $M$. vanbaalenii, leading to an increasing $D_{I}$ against the decreasing kanamycin resistance (kanamycin resistance genes in A. baylyi). Figure 3B illustrates the negative linear correlation between $D_{I}$ and the abundance of $A$. baylyi (kanamycin resistance gene abundance) within the artificial microbiotas $\left(D_{I}=\right.$ $-0.8482 \times[$ kanamycin resistance gene $]+0.7705)$. The high coefficient $\left(\mathrm{R}^{2}=0.9779\right)$ suggests a good linear regression of $D_{I}$ against kanamycin resistance. The composition of each microbiota was, therefore, calculated from the $D_{I}$ linear 
regression based on PCA-LDA, as shown in Figure 3C. The results indicated that the predicted microbial compositions had high similarity to their theoretical structure with no significant differences found $(P>0.05)$. The standard deviation of microbiota M3 (middle point in Figure 3B) was greater than the others, possibly attributing to their higher Shannon-Wiener index (1.02) than other microbiotas (0.35 to 0.94 for M1, M2, M4 and M5). Shannon-Wiener index represents the diversity of microbial community, and higher microbial diversity has been reported to increase complicated intracommunity interaction ${ }^{32}$. It might cause huge variation of microbial chemical composition, consequently leading to the difficulties in interrogating spectral biomarkers and significant standard deviation in data prediction.

\section{Quantification of kanamycin resistance dynamics within microbiota}

Figure 4A illustrates the PCA-LDA scores plot of microbiotas post-exposure to kanamycin, derived from the spectral dynamics of the artificial microbiotas (see ESI Figure S3). All the interrogated communities exhibit a dramatic shift from the original location as the exposure time increases. The M. vanbaalenii category moves towards a different direction when compared to A. baylyi and E. coli, which might be attributed to distinct cell structures between Gram-positive (M. vanbaalenii) and Gram-negative bacteria (A. baylyi and E. coli). Specifically, there is only one lipid bilayer in the membrane of Gram-positive bacteria, with a thick ring of peptidoglycan and teichoic $\operatorname{acid}^{38,39}$. On the other hand, the cell membrane of Gram-negative bacteria contains two lipid associated bilayers, which appear to increase the chance that the applied treatments influence their structure ${ }^{38,39}$. The artificial microbiotas, AM1 and AM2, follow similar trends as the A. baylyi and they come even closer to A. baylyi after extended exposure to the kanamycin antibiotic. After PCA-LDA, the most discriminating peaks were observed in Gram-negative bacteria and were attributed to lipids $\left(\sim 1750 \mathrm{~cm}^{-1}\right), v_{\mathrm{as}} \mathrm{PO}_{2}^{-}\left(\sim 1225 \mathrm{~cm}^{-1}\right)$ and $v_{\mathrm{s}} \mathrm{PO}_{2}^{-}\left(\sim 1080 \mathrm{~cm}^{-1}\right)$. Kanamycin's antimicrobial mechanism is associated with aminoglycosides, interfering with aminoacyl-tRNA recognition at the ribosomal A site and disrupting protein expression $^{40}$. Such a mechanism causes series of secondary effects, e.g., membrane damage. Our results are consistent with previous findings showing that the damage is mainly linked to a broad range of alterations associated with the elements of membranes, e.g., proteins, supported by derived peaks the protein absorbance region from 1500 to $1700 \mathrm{~cm}^{-1}$, such as Amide II $\left(\sim 1517 \mathrm{~cm}^{-1}, \sim 1543 \mathrm{~cm}^{-1}\right)$ and Amide I 
Applying the linear $D_{I}$ regression model, we successfully predicted the dynamic abundance of $A$. baylyi and kanamycin resistance within the microbiotas under kanamycin antibiotic pressures. Both artificial microbiotas, AM1 (Figure 4B) and AM2 (Figure 4C), had defined community composition at $0 \mathrm{~h}$, with $A$. baylyi (kanamycin resistance gene) accounting for $10 \%$ and $40 \%$ of the total population, respectively. Post-exposure to kanamycin, the ARGs abundance from qPCR results gradually increased to $85.0 \%$ in AM1 and $92.2 \%$ in AM2 after $12 \mathrm{~h}$, which is explained by the competitive advantages of bacteria with kanamycin resistance gene in the community ${ }^{43}$. It therefore led to a faster growth of $A$. baylyi compared to other strains and subsequent dominancy of $A$. baylyi within the microbiota. From the dynamics of discriminant functions, the predicted ARGs abundance in both microbiotas fitted efficiently with experimental data (Figure 4B and 4C). The linear correlation at each time point did not show significant difference between predicted and experimental ARGs abundance (Figure 4D), with a Pearson correlation coefficient of 0.9487 . The prediction via infrared spectroscopy coupled and multivariate analysis fitted the experimental data better at higher ARGs abundance, but was slightly lower than the qPCR results at low ARGs abundance, e.g., 33\% in Figure 4D, which might underestimate the ARGs abundance to some extent. These results not only prove that our model can be used for static community composition and abundance/dynamics of kanamycin resistance gene, but they also evaluate the impact of antibiotic pressure on kanamycin resistance gene transfer or dominancy.

(A)

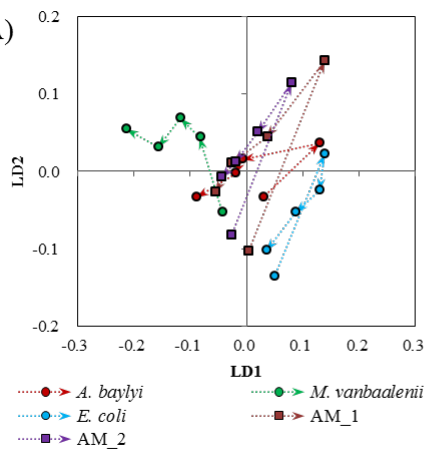

(B)

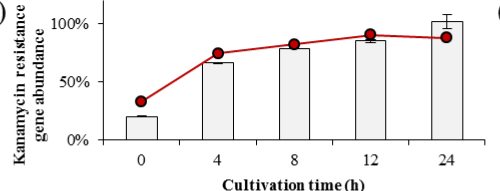

(C)

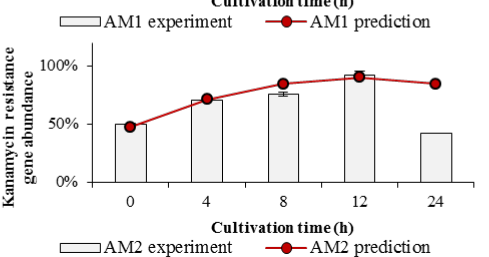

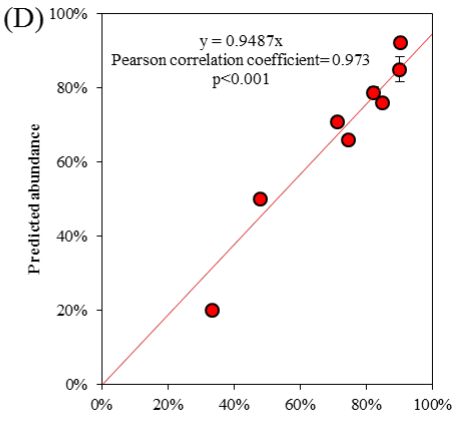

Experimental abundance (qPCR)

Figure 4. (A) Two-dimensional (LD1, LD2) scores plot after PCA-LDA of IR dynamics of artificial microbiotas. Dots along with the arrow point in each colour 
refer to the measurement at $0,4,8,12$ and $24 \mathrm{~h}$, respectively. The prediction of kanamycin resistance gene abundance is based on the dispersion among the classification groups in PCA-LDA for artificial microbiotas AM1 (B) and AM2 (C). (D) Regression correlation of kanamycin resistance gene abundance between experimental data via $\mathrm{qPCR}$ and model prediction.

It is worth mentioning that less dispersion is observed for $A$. baylyi after exposure because A. baylyi ADPWH_recA contains the kan $^{R}$ kanamycin resistance gene, which is capable of tolerating kanamycin pressure. In the present study, the $\mathrm{kan}^{R}$ kanamycin resistance gene belongs to $n p t$ encoding neomycin phosphotransferase and shows high similarity to add $A$ encoding aminoglycoside phosphotransferase (aminoglycoside kinase), which modifies the aminoglycosides by phosphoryl transfer, catalysing the phosphate addition from ATP to $3^{\prime}$-hydroxyl group ${ }^{40}$. By expressing kan ${ }^{R}$, A. baylyi ADPWH_recA inactivates the interference of protein expression by kanamycin, achieves fast recovery from suppression, and minimizes spectral alterations as compared to others. It is confirmed by the presence of consistent shifts and discriminating biomarkers in A. baylyi postexposure to kanamycin, including Amide I $\left(\sim 1630 \mathrm{~cm}^{-1}, \sim 1640 \mathrm{~cm}^{-1}\right)$ and $v_{\mathrm{as}} \mathrm{PO}_{2}^{-}\left(\sim 1222 \mathrm{~cm}^{-1}, \sim 1235 \mathrm{~cm}^{-1}\right)^{42}$.

An unexpected decline of kanamycin resistance gene was observed for AM2 artificial microbiota after $24 \mathrm{~h}$ exposure to kanamycin (42\%, Figure 4C), but the predicted kanamycin resistance by $D_{I}$ regression model remained close to $100 \%$. It might be explained by the dramatically decreasing kanamycin concentration via the metabolism of aminoglycoside modifying enzyme and the change in microbial community structure. The functions of $\mathrm{kan}^{R}$ encoding aminoglycoside kinase are stabilizing a metaphosphate transition state and inactivating kanamycin ${ }^{31}$, and the spectral alterations represent the alignment disruption of $\beta$-phosphate and $\gamma$-phosphate by amide backbone. The declining kanamycin results in less inhibition on bacteria without kanamycin resistance gene (M. vanbaalenii and E. coli), and their growth and regeneration consequently reduce the abundance of A. baylyi and $\mathrm{kan}^{R}$ gene. Alternatively, the FTIR spectral alteration reflects such phenotypic changes of the whole microbiota under the low kanamycin exposure, illustrating the fact that the majority of microbial cells within the microbiota have the pseudo-resistance to 
kanamycin. The spectrochemical interrogation therefore actually quantifies the microbial phenotypic antibiotic resistance rather than the ARGs abundance only.

Infrared spectroscopy has demonstrated the ability to diagnose the phenotypic alteration of the cellular components induced by kanamycin, hinting its potential possibility for the application to other members of the aminoglycoside family. Our findings indicate that this dispersion model coupled with PCA-LDA is a potential approach for monitoring the population dynamics within a microbiota in real-time. Additionally, the model applied in the present study summarizes the whole spectral information derived from the multivariate analysis, rather than only several biomarkers, showing its potential as a universal predicting tool for a broad spectrum of antibiotics based on well-trained databases. Though only successfully applied in the case of kanamycin through phosphotransferase resistance pathway, this technique is also feasible for detecting $N$-acetyltransferases and $O$-nucleotidyltransferases, which also belong to aminoglycoside-modifying enzymes assisted by acetyl-coenzyme A and ATP respectively ${ }^{22}$, attributing to their similar anti-kanamycin mechanisms as kan $^{R}$ encoding neomycin phosphotransferase. Future work should refer to more comprehensive range of antibiotics and their mechanisms including penicillin-class (e.g., ampicillin and amoxicillin), which disrupts the synthesis of peptidoglycan layer and inhibits bacterial cell wall synthesis ${ }^{44}$, and tetracycline, which inhibits the binding of aminoacyl-tRNA and suppresses protein expression ${ }^{45}$. For the urgent need to characterize antibiotic resistance in complex environmental microbiota with spectroscopy, the primary challenges are raised as the lack of routine protocols, reproducible computational analysis, and reliable database ${ }^{10}$. Validated in the artificial microbiota, our work provides the solutions for the first two barriers by distinguishing biomarkers representing antibiotic resistance from the numerous biological fingerprints. A well-built dataset along with robust analytical models coupled with spectroscopic methods are suggested to address the antibiotic resistance dynamics in real environmental samples.

The present study indicates that infrared spectroscopy, in conjunction with multivariate analysis, is a potential tool for diagnosing the phenotypic existence and dynamics of ARGs within microbial communities. Our work employed ATR-FTIR spectroscopy coupled with a dispersion model to quantify microbial kanamycin resistance, based on secondary derivative and PCA-LDA. This method not only 
405 quantified the static community composition of the artificial microbiotas but also

406 successfully predicted the population dynamics of microbial communities and

407 kanamycin resistance under antibiotic pressure. We also suggest that spectroscopic

408 techniques have great potential in real-time monitoring of microbiota of interest in

409 medical or environmental fields; this would provide an excellent opportunity to

410 visualize the vivid phenotypic transformation during a biological and biochemical

411 process rather than only intermittent snap-shots.

412

413 Acknowledgements N.J. was funded by Chinese Academy of Sciences and China

414 Scholarship Council. Research is supported by the Engineering and Physical Sciences

415 Research Council in F.L.M.'s laboratory (EPSRC; grant no: EP/K023349/1) and

416 National Natural Science Foundation of China in D.Z.'s laboratory (NFSC; grant no:

417 41301331). 


\section{References}

421 (1) Blair, J. M.; Webber, M. A.; Baylay, A. J.; Ogbolu, D. O.; Piddock, L. J. Nat. Rev.

422

423

424

425

426

427

428

429

430

431

432

433

434

435

436

437

438

439

440

441

442

443

444

445

446

447

448

Microbiol. 2015, 13, 42-51.

(2) Chee-Sanford, J. C.; Aminov, R. I.; Krapac, I. J.; Garrigues-Jeanjean, N.; Mackie,

R. I. Appl. Environ. Microbiol. 2001, 67, 1494-1502.

(3) Cantas, L.; Shah, S. Q. A.; Cavaco, L. M.; Manaia, C. M.; Walsh, F.; Popowska, M.; Garelick, H.; Burgmann, H.; Sorum, H. Front. Microbiol. 2013, 4.

(4) Potera, C. Environ. Health Perspect. 2013, 121, A255-A255.

(5) Smillie, C. S.; Smith, M. B.; Friedman, J.; Cordero, O. X.; David, L. A.; Alm, E. J. Nature 2011, 480, 241-244.

(6) Colomer-Lluch, M.; Imamovic, L.; Jofre, J.; Muniesa, M. Antimicrob. Agents Chemother. 2011, 55, 4908-4911.

(7) Riesenfeld, C. S.; Goodman, R. M.; Handelsman, J. Environ. Microbiol. 2004, 6, 981-989.

(8) Paraskevaidi, M.; Martin-Hirsch, P. L.; Kyrgiou, M.; Martin, F. L. Mutagenesis 2017, 32, 335-342.

(9) Kaern, M.; Elston, T. C.; Blake, W. J.; Collins, J. J. Nat. Rev. Genet. 2005, 6, $451-464$.

(10) Jin, N. F.; Zhang, D. Y.; Martin, F. L. Integr. Biol. 2017, 9, 406-417.

(11) Naumann, D.; Helm, D.; Labischinski, H. Nature 1991, 351, 81-82.

(12) Dunn, W. B.; Ellis, D. I. Trends Anal. Chem. 2005, 24, 285-294.

(13) Freedman, B. G.; Zu, T. N. K.; Wallace, R. S.; Senger, R. S. Biotechnol. J. 2016, $11,877-889$.

(14) Sun, S. W.; Wang, X. T.; Gao, X.; Ren, L. H.; Su, X. Q.; Bu, D. B.; Ning, K. BMC Bioinformatics 2015, 16.

(15) Winnard, P. T.; Zhang, C.; Vesuna, F.; Kang, J. W.; Garry, J.; Dasari, R. R.; Barman, I.; Raman, V. Oncotarget 2017, 8, 20266-20287.

(16) Li, J. Y.; Strong, R.; Trevisan, J.; Fogarty, S. W.; Fullwood, N. J.; Jones, K. C.; Martin, F. L. Environ. Sci. Technol. 2013, 47, 10005-10011. 
449

450

451

452

453

454

455

456

457

458

459

460

461

462

463

464

465

466

467

468

469

470

471

472

473

474

475

476

477

(17) Bankapur, A.; Krishnamurthy, R. S.; Zachariah, E.; Santhosh, C.; Chougule, B.; Praveen, B.; Valiathan, M.; Mathur, D. PLoS One 2012, 7.

(18) Tao, Y. F.; Wang, Y.; Huang, S.; Zhu, P. F.; Huang, W. E.; Ling, J. Q.; Xu, J. Anal. Chem. 2017, 89, 4108-4115.

(19) Siddhanta, S.; Paidi, S. K.; Bushley, K.; Prasad, R.; Barman, I. Chemphyschem 2017, 18, 72-78.

(20) Baker, M. J.; Trevisan, J.; Bassan, P.; Bhargava, R.; Butler, H. J.; Dorling, K. M.;

Fielden, P. R.; Fogarty, S. W.; Fullwood, N. J.; Heys, K. A.; Hughes, C.; Lasch, P.;

Martin-Hirsch, P. L.; Obinaju, B.; Sockalingum, G. D.; Sule-Suso, J.; Strong, R. J.;

Walsh, M. J.; Wood, B. R.; Gardner, P.; Martin, F. L. Nat. Protoc. 2014, 9, 1771-1791.

(21) Martin, F. L.; Kelly, J. G.; Llabjani, V.; Martin-Hirsch, P. L.; Patel, II; Trevisan, J.; Fullwood, N. J.; Walsh, M. J. Nat. Protoc. 2010, 5, 1748-1760.

(22) Mingeot-Leclercq, M. P.; Glupczynski, Y.; Tulkens, P. M. Antimicrob. Agents Chemother. 1999, 43, 727-737.

(23) Sadovskaya, I.; Vinogradov, E.; Li, J. J.; Hachani, A.; Kowalska, K.; Filloux, A. Glycobiology 2010, 20, 895-904.

(24) Delorenzo, V.; Herrero, M.; Jakubzik, U.; Timmis, K. N. J. Bacteriol. 1990, 172, 6568-6572.

(25) Song, Y.; Li, G.; Thornton, S. F.; Thompson, I. P.; Banwart, S. A.; Lerner, D. N.; Huang, W. E. Environ. Sci. Technol. 2009, 43, 7931-7938.

(26) Trevisan, J.; Angelov, P. P.; Scott, A. D.; Carmichael, P. L.; Martin, F. L. Bioinformatics 2013, 29, 1095-1097.

(27) Butler, H. J.; McAinsh, M. R.; Adams, S.; Martin, F. L. Anal. Methods 2015, 7, 4059-4070.

(28) Yenofsky, R. L.; Fine, M.; Pellow, J. W. Proc. Natl. Acad. Sci. U. S. A. 1990, 87, 3435-3439.

(29) Bosch, A.; Serra, D.; Prieto, C.; Schmitt, J.; Naumann, D.; Yantorno, O. Appl. Microbiol. Biotechnol. 2006, 71, 736-747. 
478 (30) Mariey, L.; Signolle, J. P.; Amiel, C.; Travert, J. Vib. Spectrosc. 2001, 26, 479 151-159.

480 (31) Wright, G. D. Curr. Opin. Microbiol. 1999, 2, 499-503.

481 (32) Flemming, H. C.; Wingender, J. Nat. Rev. Microbiol. 2010, 8, 623-633.

482 (33) Holman, H. Y. N.; Miles, R.; Hao, Z.; Wozei, E.; Anderson, L. M.; Yang, H. 483 Anal. Chem. 2009, 81, 8564-8570.

484 (34) Stewart, P. S. Int. J. Med. Microbiol. 2002, 292, 107-113.

485 (35) Hoiby, N.; Bjarnsholt, T.; Givskov, M.; Molin, S.; Ciofu, O. Int. J. Antimicrob. 486 Agents 2010, 35, 322-332.

487 (36) Karunakaran, E.; Mukherjee, J.; Ramalingam, B.; Biggs, C. A. Appl. Microbiol. 488 Biotechnol. 2011, 90, 1869-1881.

489 (37) Li, H. B.; Martin, F. L.; Zhang, D. Y. Anal. Chem. 2017, 89, 3909-3918.

490 (38) Ede, S. M.; Hafner, L. M.; Fredericks, P. M. Appl. Spectrosc. 2004, 58, 317-322.

491 (39) Morones, J. R.; Elechiguerra, J. L.; Camacho, A.; Holt, K.; Kouri, J. B.; Ramirez, 492 J. T.; Yacaman, M. J. Nanotechnology 2005, 16, 2346-2353.

493 (40) Boehr, D. D.; Thompson, P. R.; Wright, G. D. J. Biol. Chem. 2001, 276, $49423929-23936$.

495 (41) Heys, K. A.; Riding, M. J.; Strong, R. J.; Shore, R. F.; Pereira, M. G.; Jones, K. 496 C.; Semple, K. T.; Martin, F. L. Analyst 2014, 139, 896-905.

497 (42) Movasaghi, Z.; Rehman, S.; Rehman, I. U. Appl. Spectrosc. Rev. 2008, 43, $498 \quad 134-179$.

499 (43) Hibbing, M. E.; Fuqua, C.; Parsek, M. R.; Peterson, S. B. Nat. Rev. Microbiol. $500 \quad 2010,8,15-25$.

501 (44) Strominger, J. L.; Park, J. T.; Thompson, R. E. J. Biol. Chem. 1959, 234, $502 \quad 3263-3268$.

503 (45) Connell, S. R.; Trieber, C. A.; Dinos, G. P.; Einfeldt, E.; Taylor, D. E.; Nierhaus, 504 K. H. EMBO J. 2003, 22, 945-953.

505 


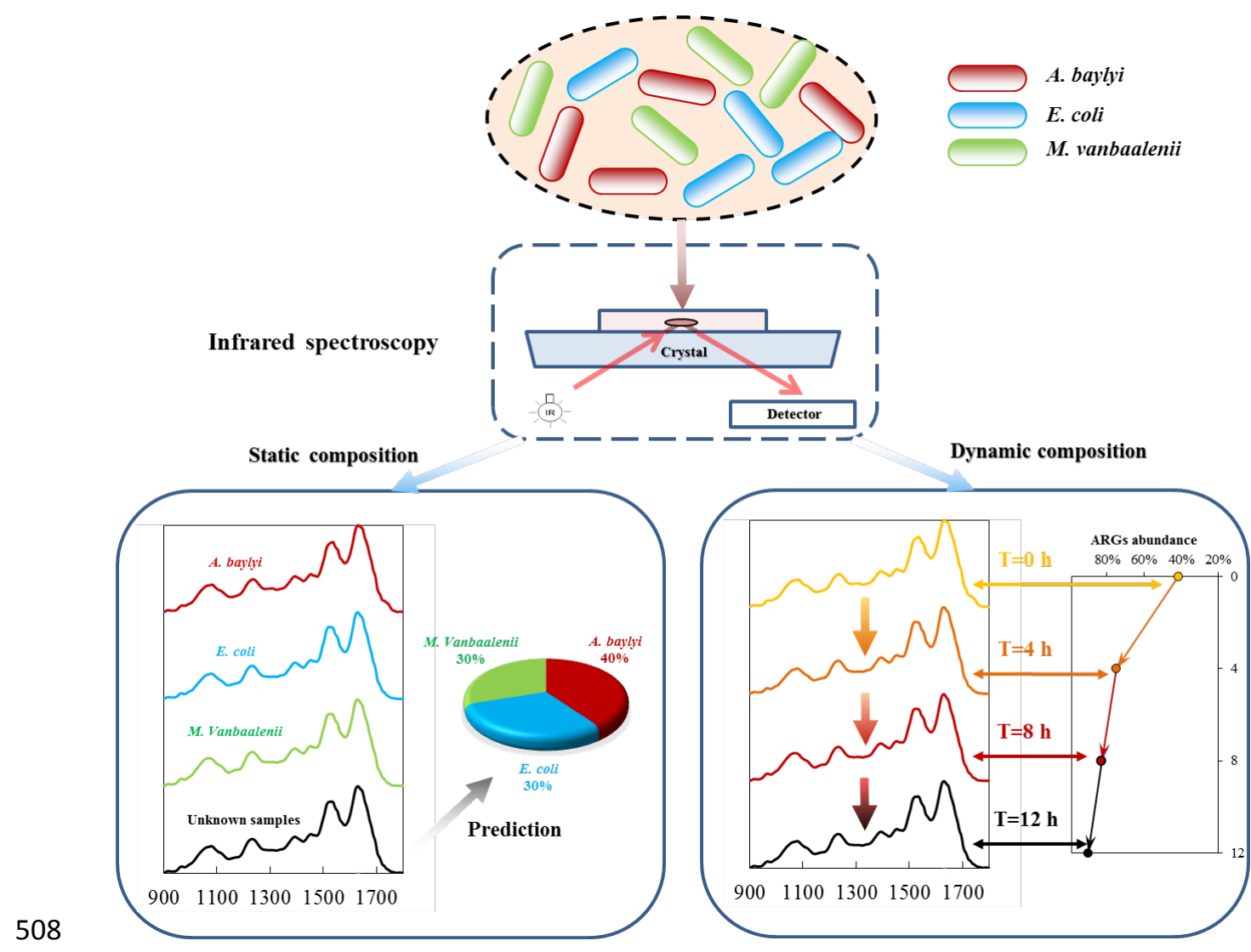

